\title{
L'hypertexte et l'avenir de la mémoire
}

\author{
Christian Vandendorpe
}

Le Débat, no 115, mai-août 2001, p. 145-155.

Selon un mythe égyptien rapporté dans le Phèdre de Platon, le dieu Thot, qui venait d'inventer l'écriture, avait présenté son invention au roi d'Égypte comme étant "un élixir de mémoire et de sagesse ". À quoi le roi avait répliqué que "cette connaissance aura pour effet, chez ceux qui l'auront acquise, de rendre leurs âmes oublieuses, parce qu'ils cesseront d'exercer leur mémoire : mettant en effet leur confiance dans l'écrit, c'est du dehors, grâce à des empreintes étrangères, non du dedans et grâce à eux-mêmes qu'ils se remémoreront les choses". Selon le roi, l'inventeur avait confondu deux choses très différentes : "Tu n'as pas inventé un élixir de mémoire, mais un moyen de retrouver un souvenir. Tu ne donnes pas la sagesse, mais l'apparence de la sagesse" (274e).

Cette histoire est surtout intéressante en ce qu'elle garde la trace du traumatisme culturel que dut représenter pour des civilisations orales la possibilité soudainement offerte par l'invention de l'écriture de créer des archives auxquelles on pourrait se référer plus tard pour retracer avec certitude et précision un événement, les termes d'un contrat ou les stipulations d'un code de lois. Dès le départ, il dut y avoir chez les Anciens, les sages de la Cité, la sensation d'avoir été dépossédés de quelque chose d'essentiel, qui était jusqu'alors l'apanage de la seule expérience. Grâce à l'écriture, de jeunes scribes pouvaient en effet accéder à un savoir complexe sans passer par les longs processus d'apprentissage et de mémorisation préalables. Bien loin d'asservir, l'écriture se révélait une technique de libération, étendant le pouvoir de l'individu bien au-delà des contingences de temps où le hasard l'avait jeté. Et l'expansion des idéaux démocratiques va de pair avec la prolifération des livres.

La puissance révolutionnaire du livre ne tient pas seulement au fait qu'il permet à un lecteur d'entrer en contact avec une pensée étrangère, mais aussi à la façon dont ce contact est mis en place, grâce au support utilisé. Pendant longtemps, l'écriture avait été perçue comme un calque de la voix, le texte devant se dérouler comme une coulée continue, destinée à être restituée par la lecture orale, laquelle était d'ailleurs souvent confiée à un esclave. Au tout début de l'écriture, le boustrophédon a même porté cet idéal de linéarité à un sommet, en faisant de la ligne de texte un fil ininterrompu allant successivement de gauche à droite et de droite à gauche, semblable au sillon tracé dans la terre par la charrue. 
Au début de notre ère, l'adoption par les premiers chrétiens du codex ou cahier cousu, en lieu et place du rouleau, constituera une révolution majeure. Régis Debray écrit à ce propos que "le christianisme a fait au monde antique de l'écrit le même coup que l'imprimerie lui fera à son tour mille ans plus tard : le coup du léger, du méprisable, du portatif." (11) En fait, par toute une série d'innovations apparemment infimes, le codex va modifier radicalement le rapport au texte. Rappelons que le lecteur devait utiliser ses deux mains pour dérouler le rouleau, et même parfois s'aider du menton pour le garder ouvert - comme le rapporte le poète Martial, dégoûté de voir les taches ainsi laissées sur le haut des rouleaux de papyrus qu'il empruntait à la bibliothèque de son quartier. Le codex au contraire est normalement posé à plat sur une table, ce qui libère la main du lecteur et lui permet de n'être plus le récepteur passif du texte, mais de s'introduire à son tour dans le cycle de l'écriture par le jeu des annotations. En outre, avec le codex, le lecteur peut accéder directement à n'importe quel point du texte. Un simple signet lui permet de reprendre sa lecture là où elle avait été interrompue, ce qui contribue aussi à transformer le rapport avec le texte. Comme le note Colette Sirat:

II faudra vingt siècles pour qu'on se rende compte que l'importance primordiale du codex pour notre civilisation a été de permettre la lecture sélective et non pas continue, contribuant ainsi à l'élaboration de structures mentales où le texte est dissocié de la parole et de son rythme. (2)

Au fil des siècles, le codex, qui repose sur l'unité discrète de la page, va mettre en place les unités logiques que sont les chapitres et les marques de paragraphes. Puis, vers la fin du Moyen Age, apparaîtront les nouveaux raffinements que sont les tables des matières et les index, grâce auxquels le lecteur peut facilement retrouver un passage donné. Avec ces divers outils de repérage d'ordre logique et visuel, le texte n'est plus conçu comme une coulée continue issue de la parole du conteur, mais comme une surface susceptible d'être appréhendée à la façon d'un tableau : le livre entre dans l'ordre du tabulaire, avec possibilité pour le lecteur d'accéder à n'importe quel segment du texte. Dès le Vle siècle, l'enluminure marque de façon éclatante le début de cette rupture avec l'ordre séculaire de l'oralité.

$\mathrm{Au}$ XVIIIe siècle, les grandes encyclopédies, en France et en Angleterre, pousseront beaucoup plus loin cet idéal d'un découpage du savoir en unités accessibles dans n'importe quel ordre, parfaitement adapté aux besoins de gens dont la mémoire et le temps sont limités. L'apparition de ce modèle coïncide avec une mutation dans les habitudes de lecture. On avait valorisé jusque là un modèle intensif de lecture, placé sous la métaphore physiologique de la digestion et de l'assimilation, voire de la rumination. C'était la lecture pratiquée dans les 
écoles et les monastères, et dont la récitation quotidienne du bréviaire par les gens d'Église constituait une sorte de modèle absolu. Dans La Nouvelle Héloïse, J ean-J acques Rousseau donnait encore comme précepte à J ulie : "Peu lire et beaucoup méditer nos lectures, ou ce qui est la même chose en causer beaucoup entre nous, est le moyen de les bien digérer". Mais cet idéal très ancien commence à être contesté, ainsi que l'ont montré les travaux de Roger Chartier et des historiens de la lecture. Les salons de lecture qui se répandent dans les pays européens au siècle des Lumières encouragent à lire beaucoup, même si c'est sous une forme superficielle et qui n'épuise pas la matière à lire. Ce modèle extensif gagnera encore en popularité au XIXe siècle, avec l'expansion massive des journaux, et au XXe siècle, avec l'extraordinaire succès des magazines. Il obtiendra finalement une reconnaissance officielle avec les programmes nationaux d'incitation à la lecture. Même la littérature finira par l'adopter explicitement avec la charte des "droits du lecteur" proposée par Daniel Pennac dans Comme un roman (1992), ouvrage qui a obtenu un vif retentissement, particulièrement dans le milieu scolaire, qui par vocation avait toujours été réfractaire à ce modèle. Des développements récents dans notre environnement textuel pousseront le modèle extensif encore plus loin, débouchant sur la lecture hyperextensive à laquelle invitent le support matériel de l'ordinateur et le format de l'hypertexte. Examinons l'histoire assez brève de ce dernier et la rupture annoncée avec l'ordre ancien.

En 1945, l'ingénieur américain Vannevar Bush, s'étant penché sur le problème du travail intellectuel, avait conçu dans son fameux article "As we may think" un système qui devrait permettre au scientifique de faire face à la quantité énorme de données et de publications à manipuler. Voulant mettre à profit les progrès de la photographie, il avait envisagé un système à base de microfiches qui permettrait de faire tenir sur une simple feuille de format standard la totalité de l'Encyclopaedia Britannica. D'après ses calculs, on pourrait ainsi emmagasiner tout le savoir humain, soit un milliard de livres, dans la caisse d'un camion. La consultation se ferait au moyen d'un memex ou unité centrale à base de fiches où seraient enregistrés les livres, revues et journaux nécessaires au chercheur, ainsi que toutes les données que celui-ci aurait accumulées, de façon à former "un supplément intime à sa mémoire". Cette machine permettrait de projeter les pages d'un livre à de très grandes vitesses. L'usager pourrait aussi faire des annotations sur les microfiches et, surtout, établir des liens entre divers éléments au moyen de références codées. Les divers liens ainsi établis constitueraient une sorte de nouveau livre où les données seraient organisées par jeu associatif, comme dans la mémoire, et que l'on pourrait ensuite réexaminer à volonté.

Le concept sera repris dans les années soixante par Ted Nelson, qui envisagera de réaliser le rêve de Bush sur ordinateur. Il forgera pour cela le concept 
d'hypertexte, qu'il tentera de concrétiser dans un réseau commercial où les éditeurs participants mettraient leur fonds à la disposition des usagers. Ce projet a été baptisé Xanadu, du nom d'un lieu mythique inventé par Coleridge dans son poème "Kubla Khan". On aurait accès à Xanadu à partir de postes de consultation installés en franchise un peu partout, tels des "McDonald de l'information". Le projet commercial ne verra cependant jamais le jour, car il sera pris de vitesse par l'arrivée du Web en 1993, au moyen duquel des serveurs connectés à l'Internet mettent leurs informations à la portée de tous.

La quantité de données stockées sur le Web croît de façon exponentielle. En décembre 2000, le nombre de pages Web accessibles au public était de un milliard quatre cents millions, selon un article de la revue Wired (8.12, p. 118), qui contenait aussi des statistiques fort éloquentes sur la place respective des diverses langues, telle qu'elle se dégage des bases du moteur de recherche Google. Ce chiffre ne représentait que la partie émergée de l'iceberg, des milliards de pages étant enfermées dans des bases privées. Pour se faire une idée de l'évolution en cours, on peut consulter un site consacré à l'archivage des sites Web et qui possède pour les quatre dernières années une collection de 14 térabits, avec un rythme de croissance de 2 térabits par mois. Ce rythme va en s'accélérant, alimenté qu'il est par des initiatives individuelles aussi bien que publiques. Ainsi, la Bibliothèque du Congrès prévoit avoir bientôt cinq millions de documents sur le Web et la Bibliothèque Nationale de France offre déjà dans Gallica plus de 15 millions de pages en ligne.

Les possibilités de stockage des données ont également connu une croissance extraordinaire au plan individuel. Nous pouvons maintenant accumuler sur nos disques durs des quantités colossales de données provenant de notre propre activité ou de celle d'autres personnes. La question est de s'y retrouver et de pouvoir garder une trace de tout ce que nous lisons ou consultons.

Sur ce dernier plan, diverses possibilités sont à notre disposition. On peut archiver ses recherches sur le Web à l'aide de signets que l'on ajoute à sa liste de favoris. Pour des données plus sophistiquées, on aura recours à des outils permettant d'enregistrer un diagramme des parcours effectués, tel Nestor, ou on regroupera dans un "livre" des fichiers texte ou des pages Web grâce à Keeboo. Depuis quelques mois, une compagnie offre d'archiver sur son site les articles qui vous intéressent, avec la possibilité d'y faire des recherches par la suite, un peu comme si on pouvait mettre à part dans une bibliothèque tous les livres que nous avons consultés afin de pouvoir plus facilement retrouver par la suite une information lue. On pourra aussi choisir de tout enregistrer sur sa machine, grâce à des disques durs de grande capacité, voire des dispositifs holographiques qui seraient bientôt capables, envisage-t-on, d'emmagasiner l'équivalent d'un milliard de livres dans un volume de la taille d'un carré de sucre. 
Même si elles relèvent d'une technologie plus raffinée, plus rapide et miniaturisée, la plupart de ces possibilités étaient déjà partie intégrante de la machine envisagée par Vannevar Bush. L'énorme avantage que le Web présente sur celle-ci se trouve ailleurs, dans une caractéristique dont cet ingénieur fasciné par les possibilités de la microphotographie n'aurait même pas pu rêver : il s'agit de l'indexation intégrale de la quasi totalité des documents accessibles sur le Web. Grâce au fait que beaucoup de données sont en format texte et que, sauf stipulation contraire ("robot excluded"), elles peuvent être colligées par des robots dans des bases de données et indexées par des moteurs de recherche, il suffit de quelques mots judicieusement choisis pour localiser une information précise parmi des millions de pages. Les internautes qui s'aventurent aujourd'hui sur le Web ne sauraient aller très loin sans ces outils que sont Google, Yahoo!, Voilà!, Copernic ou Kartoo. Grâce à eux, l'oubli des noms propres, auquel Proust a consacré des pages superbes, peut maintenant trouver un remède, ou comme disait Platon, un pharmakon. À titre d'exemple, quelqu'un qui chercherait le dialogue où Platon parle des effets de l'écriture sur la mémoire, n'aurait qu'à taper quelques mots clefs (Platon, mémoire, écriture) pour que Copernic lui donne, en première position, l'adresse de l'extrait recherché (en anglais, AltaVista produit pour les mêmes descripteurs une liste de quelque 9000 renvois, dont le cinquième mène au texte intégral du Phèdre). Bref, n'importe quel fil de mémoire, aussi ténu soit-il, permet de retrouver rapidement une information et de l'enrichir par des données nouvelles rencontrées au passage. Alors que l'ancienne civilisation orale multipliait les formules et les répétitions afin que les connaissances indispensables soient toujours présentes à l'esprit, ou du moins disponibles pour un rappel immédiat, il suffit désormais d'un mot, d'un indice infime, pour retrouver une information et, de là, ouvrir sur d'innombrables données connexes.

Bientôt, la plus grande partie du patrimoine littéraire et artistique de l'humanité sera accessible de partout, ainsi que des milliards d'articles de journaux et de revues, de traités, de notes de toutes sortes. D'ores et déjà, nombre de sociétés travaillent à perfectionner les interfaces qui permettront de puiser avec profit dans cette bibliothèque de Babel, que ce soit à partir de chez soi, sur son ordinateur, ou en marchant, à l'aide d'un organisateur personnel du genre Palm Pilot ou d'un simple téléphone. À terme, il n'est pas exclu que se développe une sorte de symbiose avec des périphériques de plus en plus furtifs. La mise au point d'ordinateurs qu'on pourrait porter comme des lunettes, intégrer au vêtement ou carrément greffer sur le corps est en train de quitter le domaine de la science-fiction pour devenir part de la réalité. Chez les spécialistes de robotique, l'utopie cybernétique profile de plus en plus son inquiétante possibilité (ㅁ). 
Au rêve ancien de tout retenir en mémoire, et de graver sur celle-ci au moyen d'une écriture intérieure, a succédé celui de pouvoir tout oublier - ou du moins de pouvoir ne retenir que superficiellement tout ce qui nous a intéressé. De plus en plus, on sait que l'on peut oublier ce qu'on voit et ce qu'on lit, parce que l'information précise restera toujours disponible et facile à retrouver dans son intégralité le jour où on en aurait besoin. Depuis déjà plusieurs années, la photographie dispensait le voyageur de s'arrêter trop longtemps à regarder les paysages traversés et permettait à des hordes de touristes de parcourir les sites du patrimoine mondial au pas de course, en se promettant de regarder plus tard les photos ou les vidéos pris à la volée. De même, les procédés d'archivage électronique nous dispensent de retenir précisément ce que nous lisons, ce qui nous permet de multiplier par cent ou par mille la quantité de données dont nous prenons connaissance ou, plus précisément, sur lesquelles nous "surfons".

Tout cela ne semble pas mettre en danger la mémoire naturelle, qui est d'abord pouvoir de reconnaître un élément déjà vu et de le rattacher à un contexte significatif. Cette fonction est inhérente au vivant et indispensable à sa préservation : pour une antilope, la capacité de reconnaître au centième de seconde les rayures d'un tigre dans les hautes herbes est une question de vie ou de mort. Lorsque cette mémoire de base est absente ou endommagée, nous avons des cas d'agnosie visuelle, comme celui de cet homme décrit par Oliver Sacks, "qui prenait sa femme pour un chapeau" et dont l'accès à la représentation était complètement barré (ㅁ) .

Ce que les nouvelles technologies sont en train de déplacer définitivement, c'est une culture où l'on valorisait la capacité de restituer intégralement et sans aide extérieure des données dont on avait pris connaissance, qu'il s'agisse d'une histoire, d'une série de formules, d'une image, etc. On sait qu'une bonne performance en matière de mémorisation exige à la fois une technique adéquate et une dépense cognitive considérable ainsi qu'un entraînement constant. Même des individus qui ont développé une mémoire prodigieuse, comme de pouvoir réciter par coeur les 24000 vers de l'Iliade, reconnaissent qu'ils doivent périodiquement rafraîchir leurs souvenirs sous peine de les voir peu à peu sombrer dans le néant. Les Anciens, qui vivaient dans une société où le livre était loin d'être omniprésent, avaient développé un art de la mémoire qui reposait sur un entraînement à associer à tout un arsenal de lieux et d'images les données à mémoriser. Frances Yates a exposé comment cette technique est apparue avec Simonide, s'est perfectionnée avec Métrodore et la Rhétorique à Herennius, pour atteindre des sommets au Moyen Age et se raffiner encore avec Ramus et Giordano Bruno (ㅁ). Mais ce furent là les derniers éclats d'un art de la mémoire qui sera bientôt discrédité. Montaigne avait déjà insisté sur la nécessité d'avoir une tête bien faite plutôt que bien pleine. Avec Descartes, ce qui importera, ce 
sera de savoir penser avec méthode. Les "monstres" de mémoire deviendront des curiosités, plutôt que des modèles à imiter, ainsi que l,a montré Harald Weinreich (므). Suivant le précepte de Rousseau qui avait interdit le "par cœeur" à son Émile, l'ancien idéal de mémorisation disparaîtra progressivement de l'école. Et ce mouvement d'élimination s'est encore affirmé au cours des trente dernières années, menaçant même les tableaux de conjugaisons et les tables de multiplication. Les exercices rituels de récitation, qui caractérisaient l'école de nos grands parents, sont devenus aussi exotiques que des récits d'anthropologues sur les mours des Trobriandais.

\section{Le fonctionnement de la mémoire}

Depuis les travaux d'Ebbinghaus et le classique ouvrage de Bartlett ( $\underline{7})$, les recherches sur la mémoire ont mis en évidence que celle-ci ne ressemble aucunement à un ensemble de tiroirs où seraient archivés des dossiers (le "colombie" des Anciens) et pas davantage à un disque dur qui aurait enregistré des traces électromagnétiques précises et fidèles des événements que nous avons vécus (la "tablette de cire"). La mémoire humaine n'est pas assimilable aux artefacts par lesquels on l'assiste. Loin d'être une simple reproduction statique, le souvenir est une opération de reconstruction cognitivement coûteuse dans laquelle intervient une part d'imagination, et qui est fonction de notre attitude envers un ensemble de données. Des recherches ont aussi montré que la façon dont un sujet raconte un événement modifie en retour le souvenir qu'il en a et que, à force de raconter une histoire inventée, un sujet peut en arriver à y croire et à se fabriquer ainsi de "vrais faux souvenirs" (ㅁ). Ce fonctionnement dynamique de la mémoire contraste avec la conception d'une mémoire entrepôt et tend à effacer la frontière que l'on croyait très nette avec l'intelligence, à l'époque d'Aristote. À lire des ouvrages de psychologie cognitive, on éprouve même parfois l'impression que l'intelligence est d'abord une façon d'utiliser sa mémoire, qui est décrite comme la "clé de voûte de l'édifice intellectuel" (99). En même temps, nul ne saurait se dissimuler que la mémoire est fragile et toujours en voie d'être altérée ou emportée par les eaux du fleuve Léthé, l'oubli mythologique des Anciens.

À partir du moment où des supports externes peuvent emmagasiner les informations qui nous intéressent et nous les redonner sur demande, il peut sembler inutile de s'en encombrer l'esprit. En libérant le cerveau humain de la nécessité d'encoder durablement des informations précises, on lui donne ainsi la possibilité de consacrer ses ressources à d'autres tâches. Les deux grands bénéficiaires de ce nouvel état de chose devraient être la capacité de généralisation et la créativité.

L'aptitude à généraliser est précieuse car elle donne à l'être humain la possibilité 
de maîtriser son environnement et notamment de faire des prédictions réussies en se basant sur les expériences passées. Cette capacité est indispensable aussi à une saine économie cognitive, faute de quoi notre mémoire serait vite submergée de détails répétitifs et encombrée d'un fatras de données inutiles, comme chez le malheureux Funes imaginé par Borges. On se souvient que ce personnage était devenu un monstre de mémoire et qu'il retenait sans effort et avec une précision totale chacune de ses perceptions, qu'il s'agisse des "formes des nuages austraux à l'aube du trente avril mil huit cent quatre-vingt-deux" ou des "crins embroussaillés d'un poulain". Tout en étant fasciné par une mémoire aussi extraordinaire, le narrateur la présente comme un effrayant handicap. Borges savait évidemment qu'une mémoire aussi vaste et précise ne pouvait exister qu'au détriment de l'abstraction et de la pensée. Comme il le dit si bien, "Penser, c'est oublier des différences, c'est généraliser, abstraire". Et il est vrai que, à partir du moment où il est libéré du souci de retenir les détails, l'esprit peut directement sauter à l'abstraction et se donner une vision plus large du réel, même si cela se fait aux dépens de la texture particulière des événements. Certes, en même temps que l'anecdote, on perd alors tout le poids du concret : l'abstraction est un incendie qui illumine l'esprit, tout en consumant son matériau.

Par ailleurs, on ne peut généraliser avec profit que si l'on a déjà construit des structures mentales d'accueil. Sans cela des heures de lecture ou de surf sur l'Internet ne laisseront pas plus de traces que les milliers d'arbres ou de rochers aperçus le long d'une autoroute à du $120 \mathrm{~km} / \mathrm{h}$. La mémorisation n'est pas un mécanisme constant, qui se déclencherait automatiquement pour tout ce qui tombe sous le coup de nos sens, comme chez le personnage de Funes. Elle ne survient que si les conditions sont favorables et pour des raisons souvent encore mystérieuses. En principe, comme le dit Roger Schank, "ce qui rend un événement mémorable dépend à la fois de son unicité et de sa signification particulière pour nous" (p. 37). Diverses stratégies aideront naturellement le sujet à retenir, comme d'associer le matériel à mémoriser à des images ou à des lieux, ou encore de l'intégrer à une histoire, car le récit serait le mode le plus fondamental de la mémoire, toujours selon Schank. Mais faut-il encore se souvenir?

\section{L'avenir de la mémoire}

Dans Total Recall (1990), film que Paul Verhoeven a produit en adaptant un récit de Philip K. Dick (10), un des personnages énonce de façon péremptoire le nouvel aphorisme par lequel se définirait l'Homme moderne - je parle de l'homo californiensis : "A man is defined by his action, not his memory". Un homme sans mémoire, c'est bien le rêve d'une société de consommation, car le flux des modes peut alors se succéder sans interruption et le murmure marchand (selon 
l'expression de l'écrivain québécois jacques Godbout) peut occuper tout l'espace. Les électrons qui scintillent à la surface de nos écrans et disparaissent dans un flux perpétuel n'ont pas de mémoire. En ce sens, ils sont les vecteurs idéaux d'une société anhistorique ou, ce qui revient au même, d'une société qui aspire à transformer le temps en simple marchandise, ainsi que l'a bien vu l'économiste américain J eremy Rifkin dans The Age of Access. On a déjà un aperçu de cette société de consommation idéale dans les groupes d'enfants et d'adolescents, que leur absence de mémoire personnelle rend parfaitement malléables aux impératifs de la mode et de la consommation de masse.

Incidemment, il est à noter que la perte de mémoire culturelle caractéristique d'une société de l'instant présent est parfaitement compatible avec l'énorme quantité d'archives secondaires qui sont créées quotidiennement, et dont la production croît plus rapidement que les archives primaires évoquées plus haut. $\mathrm{J}$ 'appelle archives secondaires toutes les traces de notre activité enregistrées à notre insu ou à notre corps défendant, et constituées par une multitude d'actes anodins que nous posons quotidiennement. Ce sont bien sûr les transactions sur carte de crédit ou de débit, qui laissent un fil de nos déplacements et de nos goûts de consommation. Ce sont aussi les communications téléphoniques et les enregistrements de caméra vidéo dans des lieux publics. À un niveau plus insidieux, il faut aussi savoir que certains logiciels de courrier électronique gardent la trace de tous les messages que nous avons reçus et envoyés, même quand nous croyons les avoir détruits.

En dépit des menaces qu'elle fait peser sur la vie privée, la mémoire informatique nous fascine pour plusieurs raisons. D'abord parce qu'elle est accessible de n'importe où, ce qui libère l'utilisateur des limitations inhérentes à un ancrage spatial. En plus de cette ubiquité, son instantanéité d'accès et son étendue potentiellement illimitée en font le réceptacle idéal du savoir humain. Enfin, elle est indexable et interrogeable de façon sophistiquée, ce qui lui a valu d'être promue du statut d'entrepôt qu'elle avait jadis à celui "d'assistant personnel". En regard, la mémoire matérielle, limitée et inerte du livre ne saurait faire le poids, surtout lorsqu'on aura réussi à mettre en place des gabarits textuels qui offriront sous mode numérique la commodité et la souplesse du codex, que ce soit sur ordinateur, avec par exemple le e-book Reader, ou sur livre électronique, ou mieux encore sur un support souple couvert d'une encre virtuelle. Avec ces nouvelles technologies du livre en pleine explosion, on peut dire qu'une page de l'histoire de l'humanité est en passe d'être tournée sous nos yeux. Et l'ébranlement dont nous sommes témoins sera au moins aussi important que le fut pour des sociétés orales l'apparition de l'écriture.

Cela dit, il est difficile de prévoir les conséquences du nouveau paradigme 
informatique. La tendance naturelle, en matière de prédiction, est de commencer par interroger l'histoire, ainsi que l'enseigna Thucydide. De fait, nous disposons des études extrêmement éclairantes au moyen desquelles Walter Ong a comparé les sociétés alphabétisées aux sociétés d'oralité primaire. Celui-ci a montré chez ces dernières l'importance, dans la production littéraire, de patrons sonores fortement rythmés, patrons qui correspondent aussi à une forme de pensée (11). Les cultures orales ont tendance à privilégier l'addition et la parataxe plutôt que la subordination, l'agrégatif plutôt que l'analytique, la redondance plutôt que la brièveté, le respect de la tradition plutôt que la créativité, la participation plutôt que l'individualisation, la pensée concrète plutôt que l'abstraction.

Les travaux d'anthropologie nous montrent ainsi qu'un changement dans notre rapport aux signes entraîne des répercussions profondes sur le rapport à la mémoire et la façon de penser, ainsi que sur l'organisation sociale. L'ordinateur va-t-il pousser plus loin la révolution apportée par l'écriture ou, au contraire, entraîner une régression ? Certains penchent pour cette dernière hypothèse, comme le suggère Mihai Nadin, pour qui nous serions à l'orée d'une Civilization of illiteracy (1997), un verdict repris par Raffaele Simone dans La terza fase (2000). Et il est vrai que l'apparition de logiciels de conversion du texte à la parole et de la parole au texte, en permettant à des individus de manipuler des symboles sophistiqués sans recourir à la langue écrite, pourra entraîner un nouveau type d'analphabétisme dans une partie de la population.

Même si de nouvelles formes de culture sont en train de voir le jour, il est toutefois douteux que l'homme de demain soit prêt à renoncer aux atouts que comporte le texte. L'écrit, en effet, tire parti du formidable pouvoir de traitement sémiotique de la vue, celui de nos sens qui possède la plus riche organisation en terminaisons nerveuses au plan du cerveau. En outre, l'écrit est par essence un média asynchrone, qui permet au scripteur et au lecteur d'y consacrer leur attention au moment qui leur convient le mieux. Il permet aussi au lecteur de choisir l'ordre dans lequel il va lire les divers passages, de régler sa vitesse de lecture et de moduler la puissance du traitement cognitif et son investissement de mémoire.

En faisant entrer dans la sphère symbolique un nombre toujours plus considérable d'activités humaines, l'ordinateur devrait hypertrophier ces caractéristiques de l'écrit, tout en favorisant aussi l'établissement d'un nouveau rapport au texte. L'immédiateté des échanges tendant à faire croire au scripteur que son destinataire partage déjà les données contextuelles dans lesquelles il est lui-même baigné, la maîtrise de l'écrit en sera rendue plus difficile et les risques d'équivoques plus nombreux. Au plan sémiotique, la capacité qu'a l'ordinateur de manipuler des images va sans doute augmenter la part de celles-ci dans les 
échanges. On observe déjà une tendance à accorder une place de plus en plus importante aux images, icônes et émoticônes, afin de compenser la froideur originelle des écrans.

D'autres mutations semblent déjà en cours, affectant la nature des histoires que nous aimons nous faire raconter et, à travers elles, notre façon de nous situer dans le temps. Le mouvement massif de rejet de la linéarité, qui a pris de l'ampleur tout au long du XXe siècle, en alimentant notamment la désaffection notée par Lyotard à l'égard des grands récits (12), pourrait avoir été encouragé par une culture lectorale qui privilégie un accès discret aux composantes d'un texte, ainsi que nous y a habitués la disposition tabulaire du journal et du magazine, et que va encore accentuer l'hypertexte. L'idéal du livre est travaillé par une aspiration qui entre en contradiction frontale avec la structure de son matériau de base, le langage, qui est essentiellement linéaire. Selon Lyotard :

Un bon livre, pour laisser être la vérité en son aberration, serait un livre où le temps linguistique (celui dans lequel se développe la signification, celui de la lecture) serait lui-même déconstruit; que le lecteur pourrait prendre n'importe où et dans n'importe quel ordre,

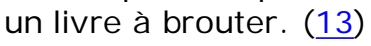

De même le lecteur d'aujourd'hui souhaite-t-il de plus en plus pouvoir lire un récit de la même façon qu'il regarde un tableau, sans être sous la dépendance de l'ordre temporel du roman traditionnel. On en arrive ainsi à valoriser les événements de sens qui se produisent par la juxtaposition de données sur un même espace, entre lesquelles le lecteur choisit à sa guise, plutôt que par mise en rapport de données organisées sur un fil temporel. Plutôt que de dérouler un fil unique à la Zola, le roman et le cinéma modernes ont appris à entrelacer des microséquences narratives formant une toile complexe et bariolée. Ce refus du linéaire serait aussi alimenté par le désir du lecteur de ne pas se trouver piégé dans un parcours de sens imposé de l'extérieur. En lui donnant le sentiment d'avoir le contrôle de la situation et de pouvoir toujours retourner aux données en jeu dans le procès de la signification, le nouvel ordre hypertabulaire favoriserait la mémoire à court terme contre la mémoire à long terme et encouragerait la fluidité et la multiplicité des contextes ouverts à des événements de sens plutôt que la saturation réitérée et obsessionnelle d'un même contexte.

L'écrit a surtout eu pour effet d'accentuer le processus d'individuation : par la lecture, un individu peut se rattacher à d'autres qui ont vécu avant lui, partager leurs émotions et assimiler leur savoir, échappant ainsi au déterminisme de l'époque qui l'a vu naître. Loin de signifier la fin de la culture comme le craignait le mythe égyptien, l'écrit a plutôt été le ferment d'un développement culturel impossible à envisager sans lui. Avec la mémoire totale offerte par l'ordinateur et le nouvel environnement créé par les technologies de communication, les 
possibilités de différenciation culturelle de l'individu ne joueront pas seulement sur une échelle verticale et temporelle, reliant le présent au passé, mais s'étendront de plus en plus à toute la surface de la planète, dans un jeu d'exploration spatiale et transculturelle permanent. Au plan des comportements sociaux, l'ordinateur va accentuer chez les usagers une tendance à adopter des identités multiples et fluides, et renforcera le désir chez chacun d'être le centre du monde, exacerbant la tendance contemporaine au narcissisme. Surtout, les couches de la population qui étaient traditionnellement exclues de la culture savante ou qui n'y avaient accès qu'à la suite d'un long processus d'acculturation verticale auront de plus en plus de lieux pour imposer leur propre modèle culturel, accentuant à leur profit la tension entre une culture horizontale centripète et une culture verticale historique. Ce mouvement est déjà commencé, surtout aux États-Unis, où il a l'appui des forces marchandes, qui mettent tout leur poids en faveur de la première.

On a souvent établi un parallèle entre le moment que nous vivons et l'avènement de la Renaissance, où l'on assiste à une expansion extraordinaire des connaissances due à l'invention de nouvelles technologies de l'information et à un début de massification de la culture. Mais les hommes de la Renaissance étaient guidés par la redécouverte de la source antique, qui était leur point de référence constant. Il n'en va pas de même aujourd'hui, où nous avons pour seul guide l'idée que nous nous faisons de l'humain et de notre destinée. Pour nourrir cette idée, cependant, nous avons à notre disposition les travaux de plus en plus nombreux de pensée prospective, ainsi que, sur un autre registre, les " mémoires du futur " imaginés par la science-fiction et dont la fonction première pourrait bien être d'éviter qu'un scénario d'avenir nous rattrape avant qu'on ait pu l'apprivoiser ou en prévoir les impasses.

\section{NOTES}

(1) Régis Debray, Cours de médiologie générale, Paris, Gallimard, p. 132.

(2) Colette Sirat, "Du rouleau au codex", dans J. Glenison, Le livre au Moyen Age, Brepols, 1988, p. 21.

(3) Voir notamment Ray Kurzweil, The Age of Spiritual machines, Viking Press, 1999. Sur l'ordinateur greffé au corps, consulter Wired, 8.02, Feb. 2000 et la réponse déjà célèbre de Bill J oy " Why the Future doesn't need us ", Wired, 8.04, Apr. 2000 (http:// www.wired.com/wired/archive/8.04/joy.html). Sur les ordinateurs "vêtements ", consulter http:// www.wearcam.org/.

(4) Oliver Sacks, L'homme qui prenait sa femme pour un chapeau, Paris, Seuil, 
1990 [1985].

(5) Farnces Yates, L'art de la mémoire, Paris, Gallimard, 1975 [1966].

(6) Sur la mise en place de ce nouveau paradigme, voir le chapitre consacré à " L'oubli éclairé ", dans Harald Weinrich, Léthé. Art et critique de l'oubli, Paris, Fayard, 1999 [1997].

(7) F.C. Bartlett, Remembering, Cambridge University Press, 1932.

(8) R. Schank et R. Abelson, "Knowledge and Memory : The Real Story ", dans R.S. Wyer, Advances in Social Cognition. Vol. VIII, Hillsdale (N.J.), Lawrence Erlbaum Associates, 1995, p. 39. Voir aussi J ean-Yves et Marc Tadié, Le sens de la mémoire, Paris, Gallimard, p. 289.

(9) A. Weil-Barais, L'homme cognitif, Paris, PUF, p. 318.

(10) Philip K. Dick, " We can remember it for you wholesale " dans The Collected Stories of Philip K. Dick, vol. 2, Citadel Twilight Book, Carol Publishing, New York, 1987, p. 35-52.

(11) Walter Ong, Orality and literacy. The technologizing of the Word, London, Methuen, 1982, p. 34-42.

(12) J ean-François Lyotard, La condition postmoderne, Paris, Minuit, 1979.

(13) J ean-François Lyotard, Discours, figure, Paris, Klinsksiek, 1971, p. 18. 